\title{
SI YO LO ESCUCHO, MIS COMPAÑER@S TAMBIÉN: EL SESGO RETROSPECTIVO EN LA INFANCIA
}

\author{
Cristina Gordo Gordo \\ Universidad de Granada. Facultad de Psicología \\ Departamento de Psicología Evolutiva y de la Educación \\ gordogordo@ugr.es \\ Sergio Moreno Ríos \\ Universidad de Granada. Facultad de Psicología \\ Departamento de Psicología Evolutiva y de la Educación
}

Recepción Artículo: 27 octubre 2021 Admisión Evaluación: 27 octubre 2021 Informe Evaluador 1: 28 octubre 2021 Informe Evaluador 2: 29 octubre 2021 Aprobación Publicación: 30 octubre 2021

\section{RESUMEN}

En este estudio evaluamos los mecanismos que subyacen al sesgo retrospectivo auditivo en escolares de entre 8 y 13 años de edad. El sesgo retrospectivo muestra el efecto que el conocimiento de un resultado posee en los juicios de los individuos. Estudios en adultos han mostrado que el sesgo en los diseños hipotéticos es producto de una atribución errónea de la fluencia generada por el conocimiento del resultado (p. ej.., "identidad de una palabra distorsionada") a las propias características del estímulo auditivo. En este estudio investigamos si estos mismos procesos de fluencia están actuando en el sesgo en la infancia. Para ello, creamos una tarea de sesgo retrospectivo auditivo en la que los escolares escuchaban varios fragmentos de canciones que parecían contener un "mensaje oculto". La tarea de los participantes consistió en estimar cuántos de un grupo formado por 6 compañeros/as de clase identificarían el "mensaje oculto" en cada canción. En una primera fase, Ios escolares realizaban sus estimaciones sin recibir información sobre el contenido del mensaje. En la segunda fase, antes de escuchar la canción y realizar sus estimaciones, los escolares eran informados del contenido del mensaje. Esta tarea incluyó una manipulación de priming por repetición que alteraba la fluencia con la que la mitad de los "mensajes ocultos" eran procesados. Los resultados mostraron un efecto modulador del priming en la magnitud del sesgo retrospectivo y sugieren que, también en la infancia, el sesgo es producto de una atribución errónea de la fluencia generada por el conocimiento del resultado.

Palabras clave: sesgo retrospectivo auditivo; escolares; priming

\section{ABSTRACT}

If I hear it, my peers hear it: hindsight bias in childhood. In this study we evaluated the mechanisms underlying auditory hindsight bias in schoolchildren between 8 and 13 years of age. Hindsight bias shows the 


\section{SI YO LO ESCUCHO, MIS COMPAÑER@S TAMBIÉN: EL SESGO RETROSPECTIVO EN LA INFANCIA}

effect that knowledge of an outcome has on individuals' judgments. Studies in adults have shown that the bias in hypothetical designs is a product of a misattribution of the fluency generated by knowledge of the outcome (e.g., "identity of a distorted word") to the characteristics of the auditory stimulus itself. In this study we investigated whether these same fluency processes are at work in bias in infancy. To this end, we created an auditory retrospective bias task in which schoolchildren listened to various fragments of songs that appeared to contain a "hidden message". The participants' task was to estimate how many of a group of 6 classmates would identify the "hidden message" in each song. In the first phase, the students made their estimates without receiving any information about the content of the message. In the second phase, before listening to the song and making their estimates, the students were informed of the content of the message. This task included a repetition priming manipulation that altered the fluency with which half of the "hidden messages" were processed. The results showed a modulating effect of priming on the magnitude of the hindsight bias and suggest that, also in childhood, the bias is a product of a misattribution of fluency generated by knowledge of the outcome.

Keywords: auditory hindsight bias; schoolchildren; priming

\section{INTRODUCCIÓN}

Uno de los sesgos más estudiados por la Psicología de la Cognición Social es el denominado sesgo retrospectivo (Fischhoff, 1975). Este sesgo muestra el efecto que el conocimiento de un resultado posee en los juicios de los individuos. Así, por ejemplo, tras ser informados de un resultado, los individuos tienden a percibir dicho resultado como más predecible de lo que éste realmente era (p. ej., Blank y col., 2008; Nestler y col., 2010) y/0 a afirmar que otras personas serían capaces de predecirlo (p. ej., Bernstein y col., 2018; Birch y col., 2017; Higham y col., 2017). Su evaluación se ha llevado a cabo principalmente a través de dos diseños experimentales: diseños de memoria y diseños hipotéticos (véase Pohl, 2007). En los diseños hipotéticos, se les pide a los participantes que estimen cuántos de un grupo formado por 100 personas conocerían, por ejemplo, la respuesta correcta a una serie de preguntas de conocimiento general (p. ej., ¿cuántos dientes tiene un mosquito?). A continuación, se les informa de la respuesta correcta a todas (o algunas) de las preguntas (p. ej., "Ios mosquitos tienen 42 dientes") y se les pide que vuelvan a estimar, pero en este caso ignorando el conocimiento recibido, el número de personas que conocerían la respuesta correcta a cada una de las preguntas.

Investigaciones sobre sesgo retrospectivo auditivo en adultos han demostrado que el sesgo es producto de una atribución errónea de la fluencia generada por el conocimiento del resultado (p. ej., identidad de una palabra distorsionada) a las propias características del estímulo auditivo (p.ej., palabra distorsionada). En concreto, el sesgo se produce porque el conocimiento del resultado facilita el procesamiento del estímulo auditivo, generando en los participantes la creencia de que "el estímulo es fácil de identificar" y que, por tanto, otras personas, pese a no disponer de la información que ellos han recibido, serían capaces de identificarlo (Higham y col., 2017). Estos estudios han hecho uso tanto de priming semántico (Higham y col., 2017) como de priming por repetición (Bernstein y col., 2018) para demostrar el papel de la fluencia en el sesgo basándose en la siguiente lógica: tanto el priming como el conocimiento de un resultado facilitan el procesamiento de un estímulo auditivo y generan la creencia de que el estímulo es fácil de identificar. Así, si el sesgo es producto de una atribución errónea de la fluencia generada por el conocimiento del resultado, incrementar previamente la fluencia con la que el estímulo auditivo es procesado a través de priming debería de reducir el efecto del conocimiento del resultado, es decir, debería de reducir el efecto de sesgo retrospectivo. Los resultados de estos estudios muestran un efecto modulador del priming en la magnitud de sesgo retrospectivo. En concreto, la magnitud del sesgo es menor en los ensayos en los que se incrementa la fluencia con la que el estímulo auditivo es procesado mediante priming (Bernstein y col., 2018; Higham y col., 2017).

\section{OBJETIVOS DE LA INVESTIGACIÓN}

En nuestro estudio aplicamos esta misma lógica para investigar los procesos psicológicos que subyacen al sesgo retrospectivo auditivo en escolares de entre 8 y 13 años de edad. Más específicamente, exploramos si, tam- 
bién en la infancia, el sesgo es producto de una atribución errónea de la fluencia generada por el conocimiento del resultado a las propias características del estímulo auditivo.

\section{MUESTRA Y/O PARTICIPANTES}

Escolares de $2^{0}$ y $3^{0}$ ciclo de la educación primaria participaron en este estudio.

\section{METODOLOGÍA Y/O INSTRUMENTOS UTILIZADOS}

Para evaluar el sesgo creamos una tarea compuesta por 6 segmentos de canciones que parecían contener un "mensaje oculto". Todas ellas eran canciones en inglés; sin embargo, estas canciones parecen contener un mensaje oculto en español. Por ejemplo, en el minuto 1.17 de la canción "De Do Do Do, De Da Da Da" del grupo británico The Police" la canción parece incluir el siguiente mensaje en español: "Bebiendo Schweppes como mejor". A los participantes se les pidió que estimasen cuántos de un grupo formado por 6 compañeros/as de clase, sin recibir información sobre su contenido, serían capaces de identificar el "mensaje oculto" de la canción.

La tarea constó de 4 fases: una fase de priming, una fase de no-información, una nueva fase de priming y una fase final de información. En las fases de priming, los escolares escuchaban un audio en el que se repetía en tres ocasiones, y en orden aleatorio, el contenido de la mitad de los "mensajes ocultos" que aparecían en las canciones a juicio. El audio fue el mismo para ambas fases de priming. En la fase de no-información, la canción era reproducida y los escolares realizaban sus estimaciones sin recibir información sobre el contenido del mensaje oculto. En la fase de información, de nuevo se volvían a reproducir las mismas canciones que en la fase de noinformación con la única diferencia de que los menores eran informados del contenido del "mensaje oculto" antes de reproducir la canción y realizar su estimación.

\section{RESULTADOS ALCANZADOS}

Los resultados mostraron que los escolares mostraron sesgo retrospectivo auditivo; es decir, afirmaron que un mayor número de compañeros/as identificarían el mensaje oculto en los ensayos en los que ellos/as habían sido informados del contenido del mensaje. Además, encontramos que el priming moduló la magnitud del sesgo retrospectivo. En concreto, el efecto de sesgo retrospectivo fue mayor en los ensayos de no-priming que en los ensayos de priming.

\section{DISCUSIÓN}

En este estudio hemos investigado los procesos psicológicos que subyacen al sesgo retrospectivo auditivo en escolares de entre 8 y 13 años de edad. Al igual que en estudios con adultos (Bernstein y col., 2018; Higham y col., 2017), encontramos que la magnitud del sesgo fue menor para los ensayos en los que el procesamiento del estímulo auditivo había sido facilitado previamente a través de priming que en aquellos ensayos en los que el procesamiento no había sido facilitado. Estos resultados sugieren que, también en la infancia, el sesgo retrospectivo auditivo es producto de una atribución errónea de la fluencia generada por el conocimiento de un resultado.

\section{CONCLUSIONES}

En este estudio exploramos un fenómeno recientemente descubierto en adultos en escolares, mostrando no sólo que el sesgo retrospectivo auditivo también opera en la infancia si no también que los procesos psicológicos implicados en el sesgo en adultos parecen ser los mismos que operan en escolares.

\section{REFERENCIAS BIBLIOGRÁFICAS}

Bernstein, D.M., Kumar, R., Masson, M. E., \& Levitin, D. J. (2018). Fluency misattribution and auditory hindsight bias. Memory \& Cognition, 46(8), 1331-1343. https://doi.org/10.3758/s13421-018-0840-6

Birch, S. A. J., Brosseau-Liard, P.E., Haddock, T. \& Ghrear, S.E. (2017). A 'curse of knowledge' in the absence of 


\section{SI YO LO ESCUCHO, MIS COMPAÑER@S TAMBIÉN: EL SESGO RETROSPECTIVO EN LA INFANCIA}

knowledge? People misattribute fluency when judging how common knowledge is among their peers. Cognition, 166, 447-458. http://dx.doi.org/10.1016/j.cognition.2017.04.015

Blank, H., Nestler, S., Von Collani, G., \& Fischer, V. (2008). How many hindsight bias are there? Cognition, 106(3), 1408-1440. http://dx.doi.org/10.1016/j.cognition.2007.07.007

Fischhoff, B. (1975). Hindsight $\neq$ foresight: The effect of outcome knowledge on judgment under uncertainty. Journal of Experimental Psychology: Human Perception and Performance, 1(3), 288-299. https://doi.org/10.1037/0096-1523.1.3.288

Higham, P.A., Neil, G.J., \& Bernstein, D.M. (2017). Auditory hindsight bias: Fluency misattribution versus memory reconstruction. Journal of Experimental Psychology: Human Perception and Performance, 43(6), 11431159. https://doi.org/10.1037/xhp0000405

Nestler, S., Blank, H., \& Egloff, B. (2010). Hindsight $\neq$ hindsight: Experimentally induced dissociations between hindsight components. Journal of Experimental Psychology: Learning, Memory, and Cognition, 36(6), 13991413. http://dx.doi.org/10.1037/a0020449

Pohl, R. F. (2007). Ways to assess hindsight bias. Social Cognition, 25(1), 1431. https://doi.org/10.1521/soco.2007.25.1.14 